\title{
APROVECHAMIENTO TURÍSTICO Y CONSERVACIÓN DEL PATRIMONIO NATURAL Y CULTURAL EN LOS PARQUES NATURALES REGIONALES FRANCESES. EL CASO DEL PARQUE DE LORENA
}

\author{
Antonio Javier LACOSTA ARAGÜÉS \\ Departamento de Geografía y Ordenación del Territorio \\ Universidad de Zaragoza
}

\begin{abstract}
Resumen: Los parques naturales regionales franceses constituyen una fórmula de protección flexible, que intenta compaginar la conservación del patrimonio natural y cultural con el desarrollo socioeconómico del territorio. La potenciación de la actividad turística juega en esto un papel fundamental. El estudio del Parque de Lorena nos permite conocer el tipo de acciones desarrolladas en su seno, así como determinar en qué grado la consolidación del hecho turístico sobre su territorio depende de esta dinámica o bien de factores externos.
\end{abstract}

Palabras clave: Parque Natural Regional, marca de calidad, flexibilidad, turismo rural, área periurbana, complejos hidrorrecreativos.

\begin{abstract}
French regional natural parks are protected in an adaptable way in order to make suitable the conservation of the natural and cultural patrimony with the socio-economic development of this area. The promotion of touristic activities is considered as a fundamental element. The study on Lorena Park let us know the type of actions developed within, as well as determine whether the importance of the internal dynamics whether external facts on its tourism consolidation.
\end{abstract}

Key words: Regional natural park, guarantee label, flexibility, rural tourism, suburban area, hydrorecreational resort.

El individuo de las sociedades desarrolladas, inmerso en una cultura recalcitrantemente urbana, busca cada vez más la distensión y el esparcimiento en el medio natural, entorno que le resulta casi ajeno. El boom de los productos turísticos que tienen en la aventura, lo rural o lo natural su razón de ser no hace sino traducir esta demanda social creciente que nace en los años setenta y se consolida en los ochenta. Los espacios naturales protegidos ejercen una especial atracción entre esta creciente clientela turística. 
No obstante, el conflicto entre las fórmulas de protección y la frecuentación turística masiva resulta inevitable. ¿Hasta dónde desarrollar el turismo, beneficioso para las economías locales, sin comprometer la riqueza natural y paisajística que constituye el principal activo de dichos espacios?

Los parques naturales regionales plantean una solución posibilista al problema. En su caso la conservación del medio no es un fin en sí mismo, como muestra la casi inexistencia de figuras de protección estricta sobre su territorio. Antes bien, este patrimonio natural y cultural de calidad se entiende como el principal activo sobre el que fundar un proyecto de desarrollo socioeconómico para el territorio. Desarrollo que pasa, forzosamente, por la potenciación de una actividad turística respetuosa pero difusa, que contribuya a revalorizar los recursos locales.

\section{EL LUGAR DE LOS PARQUES NATURALES REGIONALES ENTRE LAS MEDIDAS FRANCESAS DE CONSERVACIÓN DEL MEDIO AMBIENTE}

El gobierno francés emprendió en los años sesenta una profunda política de renovación en materia de conservación medioambiental. A las figuras de protección recogidas en la ley de 1930 (monumentos históricos, sitios naturales y reservas naturales) se añadieron los parques nacionales (1960) $)^{1}$ y los parques naturales regionales (1967). La creación del Ministerio del Medio Ambiente (1971) y 1 a integración de toda la normativa anterior en una ley de protección de la naturaleza (1976) planteó un modelo más acorde con las tendencias internacionales plasmadas en la Conferencia de Estocolmo de 1972.

Los parques naturales regionales nacieron como una fórmula de protección flexible, en contraposición al carácter estricto de los parques nacionales o las reservas naturales. En este sentido, su especificidad respecto de dichos espacios protegidos reside en tres aspectos fundamentales:

Son espacios habitados, donde la presencia humana ha modelando secularmente la naturaleza, y cuya continuidad es deseable en la medida que la conservación del territorio y el patrimonio dependen de ella.

- La conservación del patrimonio, tanto natural como cultural, no constituye un fin en sí mismo. Dicho patrimonio debe dotar al parque de una imagen de calidad y

\footnotetext{
${ }^{1}$ Con notable retraso respecto de otros países, si tenemos en cuenta que el primer parque nacional se creó en los EEUU en 1872 (Yellowstone). En Europa esta fórmula se introdujo a comienzos del s. XX en países como Suecia (1909), Rusia (1912), Suiza (1914) y España (1916).
} 
servir como base para un proyecto de desarrollo socioeconómico de la zona sustentado en la potenciación de la actividad turística y el aprovechamiento de los recursos locales.

- Los parques nacen de una iniativa local canalizada a través de la región. Todos los municipios miembros se asocian voluntariamente al proyecto del parque. Es por esto que todas las acciones que promueve deben tener un carácter negociado.

En cuanto a los objetivos que persiguen, son cuatro: proteger el patrimonio natural y cultural, a través de una gestión adaptada a la realidad de cada caso; contribuir a un desarrollo económico y social de la zona fundado en la conservación del medio ambiente, la mejora del patrimonio, el mantenimiento de la economía local y la potenciación de un turismo de calidad; promover la acogida, educación e información del público visitante favoreciendo el contacto con la naturaleza, la sensibilización hacia sus problemas y el encuentro con la cultura local; por último, realizar acciones con carácter experimental en cualquiera de los tres campos anteriores, prestando especial atención a la colaboración en programas de investigación.

\section{EL ORIGEN DE LOS PARQUES NATURALES REGIONALES}

El encargo de idear y desarrollar esta fórmula de protección fue encomendado a la D.A.T.A.R. ${ }^{2}$, quien se inspiró en las experiencias de otros países europeos (Alemania, Holanda, Reino Unido) y en los trabajos de algunos especialistas franceses sobre el tema. En 1965 se puso en marcha la primera experiencia piloto, el Parque de Saint-Amand-Raismes, cerca de Lille, y en 1966 se celebraron las Primeras Jornadas Nacionales sobre Parques Naturales Regionales en Lurs, Provenza, donde se perfilaron las ideas básicas articuladoras del proyecto: conservar la naturaleza en amplios territorios antropizados, potenciar socioeconómicamente zonas rurales en declive y dotar a las metrópolis regionales de espacios naturales de calidad que favoreciesen las prácticas recreativas de cercanía.

El 1 de marzo de 1967 se aprobó el decreto que creaba la figura de los parques naturales regionales. Su tutela quedó encomendada a la D.A.T.A.R., creándose una Comisión Interministerial con este fin. Con posterioridad, tres normativas han modificado este primer texto: el decreto de 24 de octubre de 1975, que trasfería 1 a tutela de los parques al Ministerio del Medio Ambiente y reconocía al prefecto de la región la iniciativa para su creación; la ley de descentralización de 7 de enero de 1983,

\footnotetext{
'2 La Délégation à l'Aménagement du Territoire et à l'Action Regionale (D.A.T.A.R.), organismo interministerial creado en 1963 para coordinar las líneas de trabajo de los distintos ministerios.
} 
que definía a los parques como una «estructura de planificación territorial» al tiempo que los convertía en la principal competencia de las regiones en materia de conservación medioambiental; por último, el decreto de 25 de abril de 1988, que abunda en la necesaria complementariedad entre protección y desarrollo socioeconómico.

Hasta el momento han sido creados 27 parques, uno de ellos en la Martinica. Cubren alrededor de $42.000 \mathrm{Km}^{2}$ (9 por 100 del territorio nacional) sobre 2.200 municipios y 21 regiones, albergando unos dos millones de personas. En la actualidad hay en proyecto catorce nuevos parques, uno de los cuales se localizaría en la Guayana Francesa.

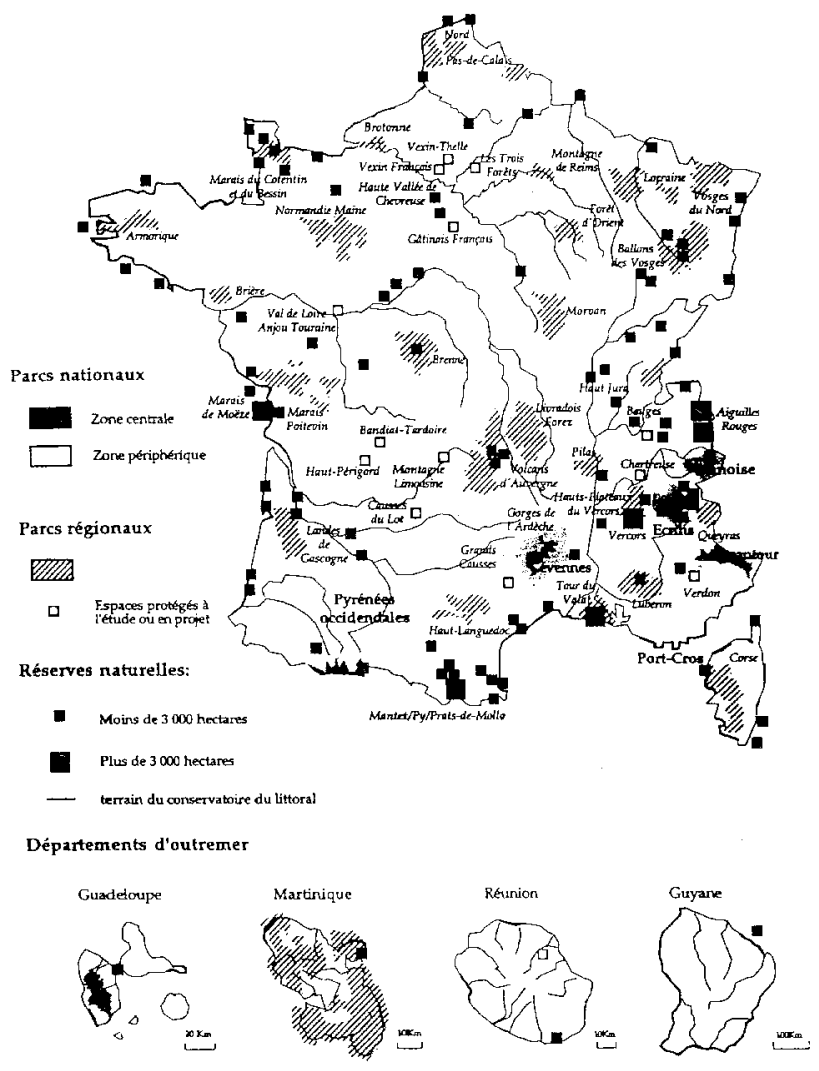

Figura 1.- Principales espacios protegidos en Francia. Fuente: D.A.T.A.R., Ministerio de1 Medio Ambiente. 


\section{CREACIÓN Y FUNCIONAMIENTO}

La iniciativa para la creación de un parque natural regional proviene de la región, previa conformidad de los muncipios que van a integrarse en él. Esta voluntad común debe plasmarse en una Carta Constitutiva consensuada por la región, departamentos, municipios y ciudades próximas al parque. En ella se fijan los límites, se marcan las prioridades y líneas de trabajo, se recogen las posibles fórmulas de financiación y se definen los órganos de gestión.

Este documento es presentado al Ministerio del Medio Ambiente quien, tras estudiar lo adecuado del proyecto, debe dar su visto bueno. El pronunciamiento favorable implica la clasificación del territorio como parque por un periodo de diez años, trascurrido el cual debe procederse a su revisión y renovación ${ }^{3}$. Esta clasificación autoriza al parque a hacer uso del logotipo "Parque Natural Regional", registrado a nombre del ministerio, que avala la calidad medioambiental del territorio con fines promocionales.

Los parques se constituyen legalmente de acuerdo con tres fórmulas jurídicas distintas: a) asociación según la ley de 1901 (caso de los parques de Ballons des Vosges y de Lorena); b) fundación (Camargue); c) sindicato mixto ${ }^{4}$ (los veinticuatro restantes). En todos los casos existen dos órganos de gestión bien diferenciados: la Dirección y el Consejo de Administración. La Dirección coordina las acciones del grupo permanente de trabajo y elabora las propuestas presupuestarias y de acción que anualmente presenta a la aprobación del Consejo de Administración. Este es un órgano colegiado con funciones consultivas y de control, que apueba las líneas de trabajo, vota los presupuestos, elige al director del parque y vela por la aplicación de la Carta; una comisión permanente se encarga de preparar las reuniones del Consejo y llevar un seguimiento de los trabajos. En el caso del Parque Natural Regional de Lorena el Consejo de Administración está integrado por el presidente de la Región, trece miembros del Consejo Regional, los alcaldes de las ciudades de Metz y Nancy, los presidentes de los tres departamentos en que se localiza el Parque (Meurthe-etMoselle, Meuse y Moselle), nueve alcaldes de municipios, el presidente del Sindicato Mixto del Lago de Madine y los presidentes regionales del Centro de Jóvenes Agricultores, la Federación de Sindicatos Agrarios, la Cámara de Agricultura, 1 a Cámara de Comercio e Industria y la Cámara del Trabajo.

\footnotetext{
3 El Parque de Marais Poitevin, a caballo entre las regiones de Poitou-Charentes y Pays du Loire, fue objeto de revisión ministerial en 1991 al consentir el drenaje de algunas zonas húmedas de gran valor ecológico para construir la autopista Nantes-Niort. Ello le supuso la retirada de la marca de calidad "Parque Natural Regional" por parte del Ministerio del Medio Ambiente. En la actualidad ha recuperado el distintivo, tras realizar las acciones pertinentes para subsanar el problema.

4 Similares a las mancomunidades aragonesas, agrupan a municipios regidos por Cartas Intercomunales de Desarrollo y Ordenación que fijan los objetivos económicos, sociales y culturales a desarrollar en común.
} 
Por lo que respecta a su financiación, los parques cuentan con dos presupuestos autónomos, uno de funcionamiento y otro de inversiones. El primero, destinado a gastos corrientes, es cubierto por los miembros del parque en la proporción asumida al, firmar la Carta. Su importe medio oscila entre los seis y ocho millones de francos, según datos de la Federación Nacional de Parques para 1993. El presupuesto de inversiones es mucho más variable, pues depende del número de proyectos emprendidos y de su envergadura. Normalmente son proyectos cofinanciados por los ministerios y colectividades territoriales implicadas. Las cantidades consignadas por el conjunto de los parques en este apartado ascendió en 1993 a 240 millones de francos.

\section{EL PARQUE NATURAL REGIONAL DE LORENA}

Creado en 1974 por la asociación de 150 municipios, en la actualidad reune doscientas mil hactáreas repartidas sobre 187 municipios de tres departamentos (Meuse, Meurthe-et-Moselle y Moselle). Dentro de su perímetro viven, según el censo de 1990 , unas 48.000 personas.

Figura 2.- Localización de la zona de estudio.

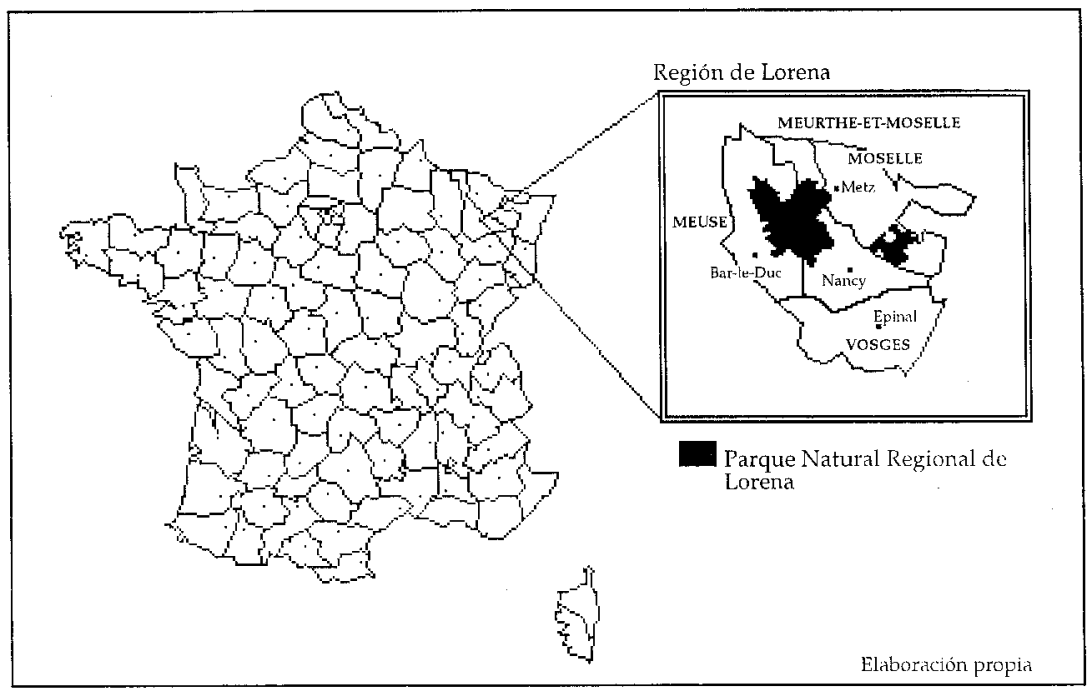


El Parque está dividido en dos partes, una a cada lado del valle del Mosela. Sobre esta vía de comunicación natural se organiza el sistema urbano regional (aglomeraciones de Nancy, Metz y Thionville), gracias a una densa red de transportes (tren regional METROLOR, autopista exenta de peaje A-31, carretera nacional RN-57, canales fluviales). Es una zona densamente urbanizada, especializada en industrias pesadas ligadas a la producción metalúrgica y energética.

La parte occidental del Parque se localiza sobre una zona de relieves estructurales, las cuestas del Mosa y del Mosela, que separan las cuencas de ambos ríos. En el centro se encuentra la depresión arcillosa de la Petite Woëvre. Cada una de estas unidades presenta conjuntos paisajísticos bien diferenciados: campos de cereal y bosque de hayas en los reversos de las cuestas; prados, bosques de robles y estanques en el fondo de la depresión; cultivos de frutales y viñedo en el bien soleado frente de la cuesta del Mosa; por último, espacios fuertemente urbanizados en el frente de la cuesta del Mosela, ya en el exterior del Parque, dada su mayor inserción en el sistema urbano regional.

En esta zona los elementos más interesantes del patrimonio natural son el estanque de Lachaussée (lámina de agua artificial protegida por el Convenio RAMSAR dada su importancia para las aves migratorias), el Bosque de la Reina (robledal salpicado de estanques, vestigio de los bosques de aprovechamiento comunal en el dominio agrario del openfield) y los cursos fluviales obsecuentes de los ríos Ruptde-Mad y Esche, que atraviesan la cuesta del Mosela. Del patrimonio cultural conviene destacar abundantes monumentos históricos (acueducto romano de Ars-surMoselle, iglesias románicas y góticas fortificadas, castillos de origen medieval como los de Dielouard, Hattônchatel y Jaulny); los elementos ligados al pasado bélico de la región, especialmente de la Primera Guerra Mundial (fortificaciones militares de Troyon y Génicourt; cementerios de campaña de Gravellotte, Thiacourt y Andilly; monumento conmemorativo de Montsec); y algunos núcleos urbanos de gran interés (conjunto medieval de Hattônchatel; pueblos-calle típicos de la Lorena como SaintJulien-sous-les-Côtes o Giravousin; pueblos vitivinícolas de las Cuestas de Toul como Bruley, Lagney o Lucey).

En cuanto a la mitad oriental, ocupa una zona llana drenada por el río Seille. En esta parte se alternan praderas, bosques de hayas y robles, así como numerosos estanques. Entre ellos destacan por sus dimensiones los de Lindre, Mittersheim y Gondrexange (todos artificiales). En el mismo valle del Seille se localizan algunas saladas de gran valor ecológico, producto de la precipitación de sales en medios lagunares endorreicos. En cuanto al patrimonio cultural, destacan algunos conjuntos urbanos por su trazado medieval (Vic-sur-Seille, antigua sede episcopal), militar (Marsal, fortaleza del s.XVII en forma de estrella), tradicional (pueblos-calle de 
Omerray o Mittersheim, entre otros) o simplemente pintoresco (Tarquimpol, sobre una pequeña península del estanque de Lindre).

\section{LA GESTIÓN DEL PARQUE}

Al abordar este tema conviene tener presente que las limitaciones reglamentarias y financieras condicionan en alto grado las posibilidades de acción de los parques. No en vano, éstos actúan generalmente como promotores de los proyectos, siendo organismos públicos o agentes privados quienes los llevan a efecto.

El ejemplo más claro de este funcionamiento lo encontramos en el campo de la protección, labor que los parques desarrollan de forma diversa, recurriendo en última instancia a figuras de protección estricta. Sobre sus territorios rigen las reglamentaciones clásicas en materia de caza, pesca y montes, así como las específicas para la conservación del patrimonio artístico, siendo responsables de su aplicación los organismos competentes (O.N.F. ${ }^{5}$, D.R.A.C. ${ }^{6}$, etc). Si la conservación de un elemento patrimonial se hace necesaria, el parque se encarga de promover la figura de protección adecuada ante la instancia pertinente (Ministerios del Medio Ambiente o de Cultura, Prefecto de la Región, Conservatoire des Sites Lorrains, etc), siendo éstas quienes se encargan de ponerla en pie. En el caso del Parque de Lorena tan sólo existen cinco figuras de protección estricta en relación con la naturaleza, todas ellas de carácter menor: tres decisiones prefecturales para la protección de biotopos (nacimiento y afluente del río Seille, nacimiento del río Gorze), un paraje natural inscrito (el estanque de Lachaussée) y, por último, un paraje natural clasificado (el tramo medio del río Esche).

Frente a estas soluciones extremas, los parques suelen desarrollar su labor de conservación por otros cauces: la investigación, la sensibilización de los habitantes, el control de las nuevas actividades que se implantan sobre su territorio y, por último, las actuaciones para recuperar medios naturales, paisajes tradicionales o elementos culturales significativos.

En el ámbito de la investigación, el personal del Parque trabaja en la realización de inventarios que permitan conocer la riqueza patrimonial de su territorio para actuar de la forma más adecuada ante cualquier problema que pueda plantearse. Para ello colabora con organismos públicos (Universidad, ONF, Conservatoire des Sites Lorrains, etc) y privados (asociaciones ornitológicas, culturales, etc). En este sentido su

\footnotetext{
5 O.N.F. (Office National des Fôrets).

${ }^{6}$ D.R.A.C. (Direction Régionale des Affaires Culturelles).
} 
integración en los programas Z.I.C.O. ${ }^{7}$ y Z.N.I.E.F.F. ${ }^{8}$ son sus trabajos más ambiciosos. Entre 1980 y 1993 el $63 \%$ de los municipios del Parque había recibido alguna de estas labores de inventariado, permitiendo conocer la existencia de 75 especies vegetales raras en la región, 4 de ellas muy raras en Francia.

La sensibilización de los habitantes hacia los problemas del Parque con vistas a que sean ellos mismos los principales garantes de la conservación del territorio, es in objetivo clave del equipo gestor. Los esfuerzos se canalizan hacia dos problemas fundamentales, las prácticas agrícolas nocivas y la proliferación de nuevas construcciones. Respecto a las primeras, se imparten cursos de formación y realizan proyectos encaminados a la recuperación de cultivos y medios naturales, como veremos más adelante. En cuanto al control de la actividad inmobiliaria, el parque desarrolla desde 1993 una experiencia pionera que, de probarse eficaz, podrá extenderse a 1 conjunto de la región: la creación de un Atlas Municipal donde se recogen, entre otros niveles de información, los elementos naturales o humanos con valor patrimonial y la división del término municipal en distintas zonas según su calidad paisajística. Para su confección se utilizan las bases de datos geográficos generadas a partir de los inventarios. Con este atlas se pretende dotar a todos los ayuntamientos miembros del Parque de un instrumento de referencia que les ayude en la toma de decisiones, a fin de que conozcan en todo momento su alcance real, especialmente en el caso de la concesión de licencias de obra. Este trabajo adquiere especial significación tras la aprobación de la ley del 8 de enero de 1993 sobre la protección y valoración de los paisajes, ya que obliga a que los documentos de urbanismo (Esquemas Directores de Ordenación, P.O.S. ${ }^{9}$ y otros) de las colectividades territoriales firmantes de la Carta estén de acuerdo con las orientaciones establecidas por el parque.

En cuanto al control de nuevas actividades sobre su territorio, el Parque, al no poder imponer limitaciones restrictivas, asesora sobre la mejor forma de llevarlas a cabo respetando los principios fijados en la Carta. Hasta el momento, los casos más significativos los constituyen los complejos hidrorrecreativos construidos en su interior y que analizaremos en la tercera parte del artículo.

\footnotetext{
7 Z.I.C.O. (Zone d Tntérêt Communitaire pour les Oiseaux). programa de inventariado iniciado en 1980 como aplicación de la directiva comunitaria 79/409 CEE para la protección de aves salvajes. En España sus homólogos son las Z.E.P.A. (Zonas de especial protección para las aves).

8 Z.N.I.E.F.F. (Zone Naturel d'Intérêt Ecologique, Faunistique et Floristique. Programa de alcance nacional iniciado en 1983 por el Ministerio del Medio Ambiente, destinado a crear una gran base de datos sobre los hábitats naturales, especialmente de los más amenazados.

9 P.O.S. (Plan d Ocupation des Sols). Son los homólogos franceses a los Planes Generales de Ordenación Urbana españoles.
} 
Por último, el parque promueve proyectos para recuperar medios naturales, paisajes tradicionales o elementos culturales significativos que repercutan en la mejora del conjunto del territorio. Las actuaciónes llevadas a cabo con este fin son múltiples, y van desde la adquisición de una antigua cantera para la protección de una formación vegetal característica (césped calcáreo de Génicourt) hasta le mejora del entorno de pueblos pintorescos (Hattonchâtel, Vic-sur-Seille, Marsal, Tarquimpol), pasando por la recuperación de cultivos tradicionales abandonados (la ciruela de la variedad mirabelle en el frente de la cuesta del Mosa o los viñedos en el frente de las Cuestas de Toul), la mejora de medios naturales aprovechados por el ganado (praderas halófilas del Valle de la Seille) o la conservación de edificios típicos (architecture ì colombages, casas con muros de argamasa y estructura de madera a la vista).

Junto a esta faceta de protección adaptada, encontramos las acciones relacionadas con la dinamización socioeconómica del territorio. Se orientan en dos direcciones básicas: fomentar la frecuentación turística y potenciar actividades económicas tradicionales.

La promoción del Parque se dirige a dos tipos de clientes bien definidos: los visitantes en general y los niños y jóvenes. En todos los casos el planteamiento didáctico es muy acusado. Para los visitantes en general el Parque ha editado folletos; señalizado senderos; creado centros de interpretación (Maison des oiseaux, en Lachaussée; Centro para la interpretación del paisaje, en Hattônchatel; Centro de interpretación del Estanque de Lindre); organizado salidas guiadas al campo; celebrado grandes eventos culturales (festivales medievales) o creado una red museográfica. Con estas acciones, se ha conseguido captar una clientela no muy numerosa, pero de gran importancia por su reparto difuso sobre el territorio. El caso de los ocho museos del Parque es el más significativo: por sus salas pasan anualmente una media de 40.000 visitantes, cifra modesta (tan sólo el 4 por 100 de las entradas a los museos loreneses) pero significativa dada su localización en núcleos rurales de pequeño tamaño. En cuanto al segundo tipo de clientes, los niños y jóvenes, constituyen un dominio de acción prioritaria para el Parque, que colabora con el Ministerio de Educación y la D.R.A.C. organizando charlas en colegios, visitas a museos y centros de interpretación así como en la organización de campamentos sobre temas vinculados a 1 medio ambiente y el patrimonio.

Respecto a la potenciación de nuevas actividades económicas, las experiencias más interesantes se han desarrolladó en la recuperación de prácticas tradicionales como la elaboración de productos artesanos (licores y frutos secos elaborados a partir de la mirabelle, vinos, etc) o la explotación piscícola de ciertos estanques (producción de carpas en el estanque de Lachaussé, gestionada por un centro para la integración de disminuidos físicos). También se han creado centros para su comercialización, fomentando el asociacionismo de los productores (Le Verger Conservatoire, en 
Hattonchatel, y el Centro Côtes du Toul, en Bruley). Además, el parque ha trabajado como asesor de algunas iniciativas turísticas: la creación de albergues (el Carrefour des Jeunes, en Beaumont, y la Maison de l'Artisanat, en St.-Maurice-sous-les-Côtes), Ia remodelación de camping o la creación de pequeños complejos hidrorrecreativos.

Como se observa, las iniciativas fomentadas por el Parque en relación con el turismo y los sectores productivos se adecuan a su pretensión de revalorizar los recursos locales dando a conocer su patrimonio natural y cultural. Estas acciones, que consolidan su imagen como espacio de calidad medioambiental, no bastan sin embargo para explicar toda la dimensión del hecho turístico sobre su territorio, ya que están dirigidas bien para excursionistas bien para una clientela cualificada pero poco numerosa.

\section{EL HECHO TURÍSTICO SOBRE EL TERRITORIO DEL PARQUE}

La labor desarrollada por el Parque explica sólo parcialmente el desarrollo de la actividad turística sobre su territorio. Junto a esta acción dinamizadora, intervienen factores externos que explican en mayor grado aspectos tales como la localización de alojamientos o la jerarquización de los flujos turísticos que convergen sobre el territorio. En concreto, conviene destacar tres de ellos: a) la existencia de una importante clientela potencial; b) la atracción ejercida por los grandes complejos hidrorrecreativos establecidos en su perímetro; c) la proliferación de residencias secundarias.

a) La posición junto a la metrópoli Nancy-Metz, al sur de la región industrial que aglutina las cuencas siderometalúrgicas de Lorena, Luxemburgo y el Sarre alemán, convierte al Parque en destino turístico privilegiado. Sin ir más lejos, éste fue uno de los factores determinantes para la construcción del complejo hidrorrecreativo del Lago de Madine: según un estudio del O.R.E.A.M. ${ }^{10}$, el o3\% de la población lorenesa residía en 1975 a menos de hora y media de desplazamiento; las aglomeraciones de Nancy y Metz, principales centros urbanos de la región, quedaban comprendidas dentro de la isocrona de una hora de desplazamiento.

\footnotetext{
10 O.R.E.A.M. (Organisme d'études et d'aménagement d'aire metropolitaine). Creados por la D.A.T.A.R. en 1966, son uno más de los instrumentos que esta institución puso a disposición de las grandes aglomeraciones urbanas francesas, en especial, París y las ocho "Metrópolis de Equilibrio" (Burdeos, Estrasburgo, Lille, Lyon, Marsella, Nancy-Metz, Nantes-Saint Nazaire y Tolosa), para controlar y organizar su crecimiento.
} 
El estudio de la procedencia de los turistas que acuden a Lorena corrobora tanto la estrecha dependencia de la demanda endógena, como el importante peso de los turistas extranjeros provenientes de países vecinos. Según una encuesta regional realizada en el verano de 1994 por el IL2E ${ }^{11}$, el 31 por 100 de los encuestados eran loreneses, seguidos por alemanes (13 por 100), holandeses (9 por 100), franceses de las regiones vecinas especialmente de Champagne-Ardenne (9 por 100) y belgas (6 por 100). Sin embargo, el análisis del peso ejercido por las distintas clientelas en su lugar de origen, de acuerdo con el método propuesto por CHADEFAUD (1971) 12, plantea alguna matización. La primera hace referencia a la clientela luxemburguesa, poco importante en cifras absolutas pero una de las principales en términos relativos junto a holandeses, belgas y franceses de las regiones próximas. La segunda, más llamativa si cabe, alude a la clientela alemana, segundo grupo turístico en importancia: su escaso peso en el país de origen sitúa a Lorena como un destino secundario, interesante a escala regional, pues son los alemanes de la región del Sarre quienes acuden atraídos por el fuerte tropismo que implica el cambio de país así como por unas condiciones económicas e inmobiliarias más favorables.

b) La existencia de láminas de agua acondicionadas con fines recreativos, lo que lo franceses denominan plan d'eau, ${ }^{13}$ está en el origen de los principales flujos turísticos que convergen sobre el territorio del Parque. La vocación turístico-recreativa de estos espacios arranca, según ha apuntado REPARAZ (1991), bien de la reconversión funcional de antiguas retenciones bien de la creación ex nihilo para atender una demanda creciente. En el Parque encontramos tres tipos de complejos hidrorrecreativos: pequeños complejos intensivos, grandes complejos espontáneos y grandes complejos planificados.

Los pequeños complejos intensivos nacen de la recuparación de estanques para su aprovechamiento turístico-recreativo. En el Parque existen tres complejos de este tipo, localizados en su parte occidental: la Base de loisirs Les Epichées, en Sommedieue, la Base de loisirs du Col Vert, en Bonzée, y el Centre Départamental de Plein Air, en SaintMihiel.

\footnotetext{
11 IL2E (Tnstitut Lorraine d'Etudes et d’Evaluation des Politiques Publiques).

12 El índice de Chadefaud permite ponderar la atracción ejercida por un determinado destino turístico en los focos emisores. Se formula de la siguiente forma: $\mathrm{R}=(\mathrm{Tij} / \mathrm{Pi}) \cdot 10^{5}$; siendo $\mathrm{Tij}$ el número de turistas de procedencia j contabilizados en el destino i y Pj la población total del foco emisor j. El resultado se expresa en tantos por cien mil.

13 La denominación correcta, más técnica si se quiere, es la de base de plein air et loisirs. Este nombre fue acuñado por la Secretaria de Estado para la Juventud y el Deporte en circular del 20-I-1964, e intentaba dar carta de naturaleza al fenómeno todavía incipiente de los complejos recreativos situados en áreas periurbanas, especialmente alrededor de lagos.
} 
Figura 3.- Origen espacial de los turistas que veranean en Lorena

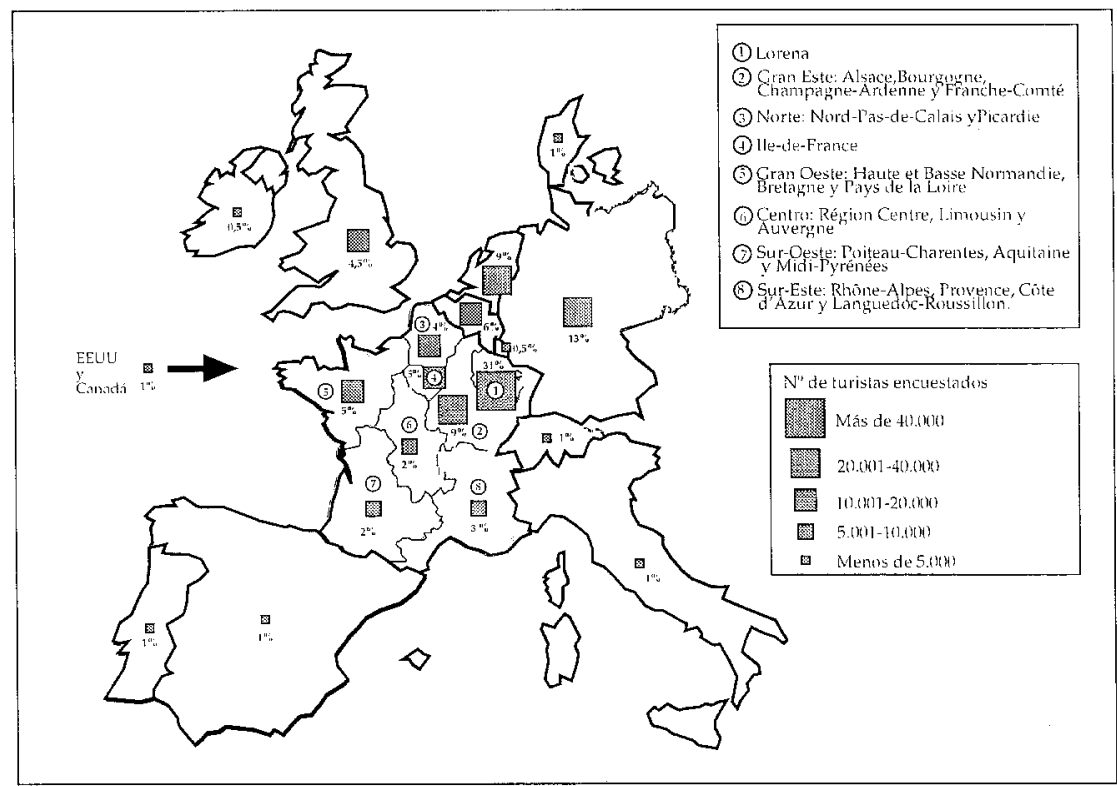

Fuente: Enquete de Clienteles realisée pendant l'été 1994. IL2E. Elaboración Propia.

Figura 4.- Peso de la clientela turística lorenesa en su lugar de origen, según el índice de Chadefaud

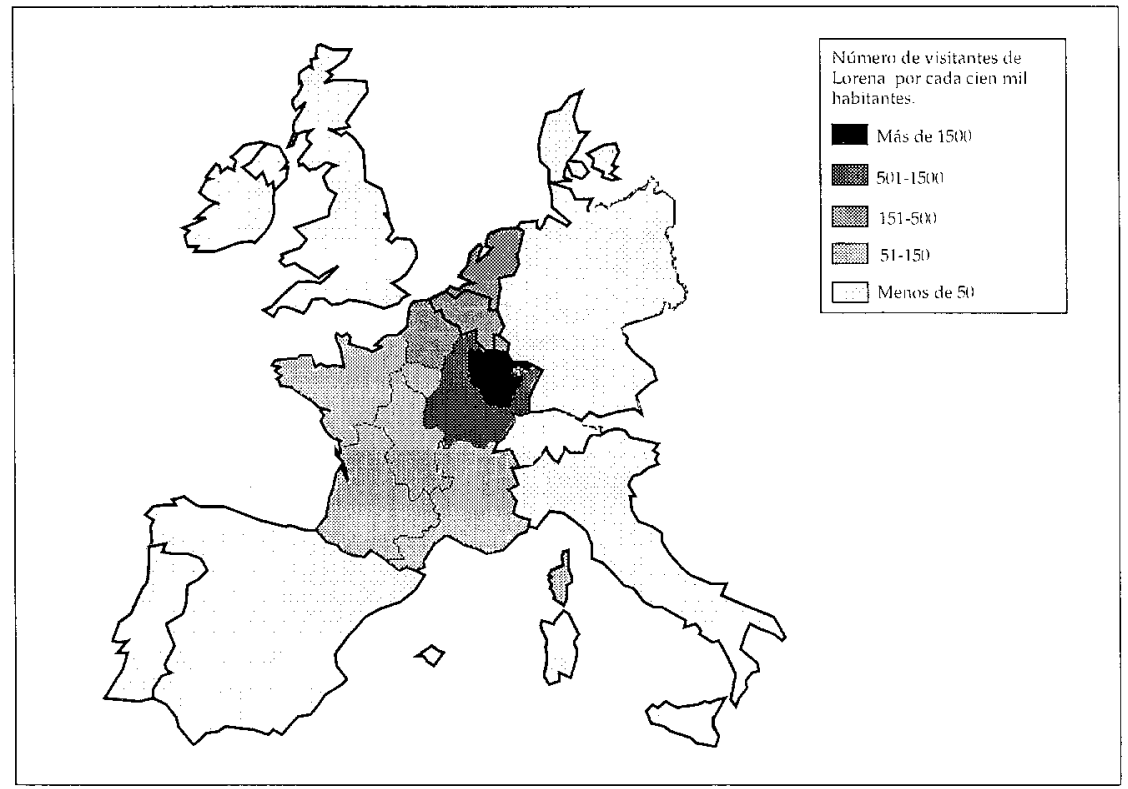

Fuente: Enquete de Clienteles realisée pendant l'été 1994. IL2E. Elaboración Propia. 
En todos los casos citados su morfología es similar: espacio reducido (menos de 50 hectáreas); perímetro vallado para controlar el acceso de visitantes; estanque de pequeño tamaño (hasta 10 hectáreas); orillas acondicionadas para la práctica recreativa (baño, pesca, iniciación a deportes acuáticos); pistas deportivas; posibilidad de alojamiento (camping); por último, un mínimo de servicios complementarios (restaurante, bar, supermercado, sala de fiestas). Su capacidad de atracción es importante: el centro de Bonzée recibió en 1994 un total de 48.000 visitantes.

Los grandes complejos de origen espontáneo se localizan en el límite oriental del Parque, aunque fuera de su perímetro. Se trata de los estanques de Mittersheim, Stock y Gondrexage, construidos en el s. XIX para permitir la navegación fluvial entre las cuencas siderometalúrgicas del Sarre alemán y la Lorena. No nacen, como en el caso anterior, de un proyecto planificado sino de una dinámica turística espontánea que tiene su origen en la atracción del agua. Su manifestación espacial más evidente es 1 a proliferación desordenada de residencias secundarias sobre las riberas de los lagos, dando lugar a una morfología característica: un gran lago artificial (cientos de hectáreas); terrenos próximos a la orilla fragmentados en parcelas de pequeño tamaño; predominio de viviendas de tipo precario (caravanas, casas prefabricadas); algunas zonas residenciales de alto nivel; e inexistencia de grandes equipamientos colectivos.

En cuanto a los complejos turísticos planificados, el único que existe dentro del Parque es el de Madine. Fruto de la iniciativa pública, se construyó con la doble finalidad de aprovisionar de agua la ciudad de Metz y crear un centro que polarizase las demandas recreativas de la aglomeración Nancy-Metz. Abierto al público en 1979, los sucesivos planes de inversión han dotado al complejo de una importante oferta recreativa (playas artificiales, puerto deportivo, campo de golf, centro ecuestre, escuela de vela, pabellones polideportivos), servicios (restaurantes, bares, supermercados, alquiler de eqipos, sala de fiestas) y alojamiento (campings, albergue, village de vacances). Su morfología contrasta con el caso anterior en dos aspectos básicos: la existencia de grandes eqipamientos colectivos y la ordenación de espacios según su funcionalidad Todo ello, sumado a la profundización en las actividades educativo-recreativas, permitió que el complejo recibiera en 1994 unos 300.000 visitantes y 24.000 pernoctaciones. El funcionamiento de este complejo se organiza como un Sindicato Mixto que agrupa a las colectividades territoriales implicadas (región, departamento, municipios). El Director del Parque está representado en su Consejo de Administración.

c) La residencia secundaria constituye la principal oferta de alojamiento dentro del parque. Entre 1962 y 1991 su crecimiento ha sido continuo, pasando de 752 a 2.237 viviendas. En términos relativos, y considerando únicamente los municipios rurales, este crecimiento ha sido más acusado en el Parque que en el conjunto regional: un 298 por 
100 frente a un 251 por 100 para dicho periodo, observándose un cierto declive a partir de 1975. En conjunto, unas 11.000 camas turísticas aproximadamente.

El cálculo de la tasa de función residencial14, empleada entre otros por BARBIER (1966) y RENUCCI (1984), permite extraer una serie de explicaciones verosímiles sobre la evolución espacial y temporal del fenómeno (Fig. 5). En primer lugar, se aprecia una acusada tendencia a la concentración en aquellos puntos que ejercen mayor atracción turística, bien por su funcionalidad recreativa (lagos de la Madine, Mittersheim, Stock y Gondrexange) bien por su belleza paisajística (cuesta del Mosa, ríos Rupt-de-Mad y Esche ). En segundo lugar, se observa que los municipios próximos al corredor del Mosela y a ciudades menores como Toul, Commercy, SaintMihiel y Verdun han visto decrecer sı tasa de función residencial a partir de 1975, consecuencia de dinámicas periurbanas que se han traducido en la conversión de viviendas secundarias en principales.

En relación con estos factores, resulta interesante estudiar el volumen y localización de la oferta de alojamientos turísticos dentro del Parque (hostelería, camping y vivienda de turismo rural), pues a falta de datos fiables de frecuentación son la mejor aproximación a la plasmación del hecho turístico sobre el territorio. La hostelería vinculada al Parque contaba en 1994 con 48 establecimientos y un total de 981 habitaciones. El 40\% de éstas correspondia a hoteles y el 60\% restante a hotelesrestaurantes. El tamaño medio de los establecimientos era de 20 habitaciones, sensiblemente inferior a los de las principales ciudades regionales (55 en Metz y 38 en Nancy). En cuanto a su distribución espacial, el 79\% se localiza en las ciudades-puerta del Parque, destacando Verdun, centro turístico vinculado a la Primera Guerra Mundial (su Centro Internacional para la Paz recibe 400.000 visitantes al año) y Pontà-Musson, pequeña ciudad industrial beneficiada de su posición central entre las capitales regionales (aeropuerto, futura estación del TGV). El $21 \%$ restante se localiza en el interior del Parque, siendo todos ellos hoteles-restaurantes, especialización fácilmente explicable por la importancia del grupo de excursionistas entre los visitantes.

Los camping ofertaban en 1994 un total de 2.102 plazas repartidas entre dieciseis establecimientos. Su tamaño medio era de 131 plazas, muy superior a la media regional situada en 81 . El $69 \%$ está vinculado a los complejos hidrorrecreativos citados. Destacan las 600 plazas en el Lago de Madine y las 300 del Lago de Mittersheim (Fig. 6).

\footnotetext{
$14 \mathrm{La}$ tasa de función residencial se formula de la siguiente forma: $\mathrm{TFR}_{\mathrm{i}}=\mathrm{RS}_{\mathrm{i}} \bullet 100 / \mathrm{RP}_{\mathrm{i}}$; siendo $\mathrm{TFR}$ la tasa de función residencial de la localidad $i, R_{i}$ el número de viviendas secundarias en dicha localidad y $R P_{i}$ las viviendas principales.
} 
Figura 5.- Evolución de la residencia secundaria en el Parque Natural Regional de Lorena.
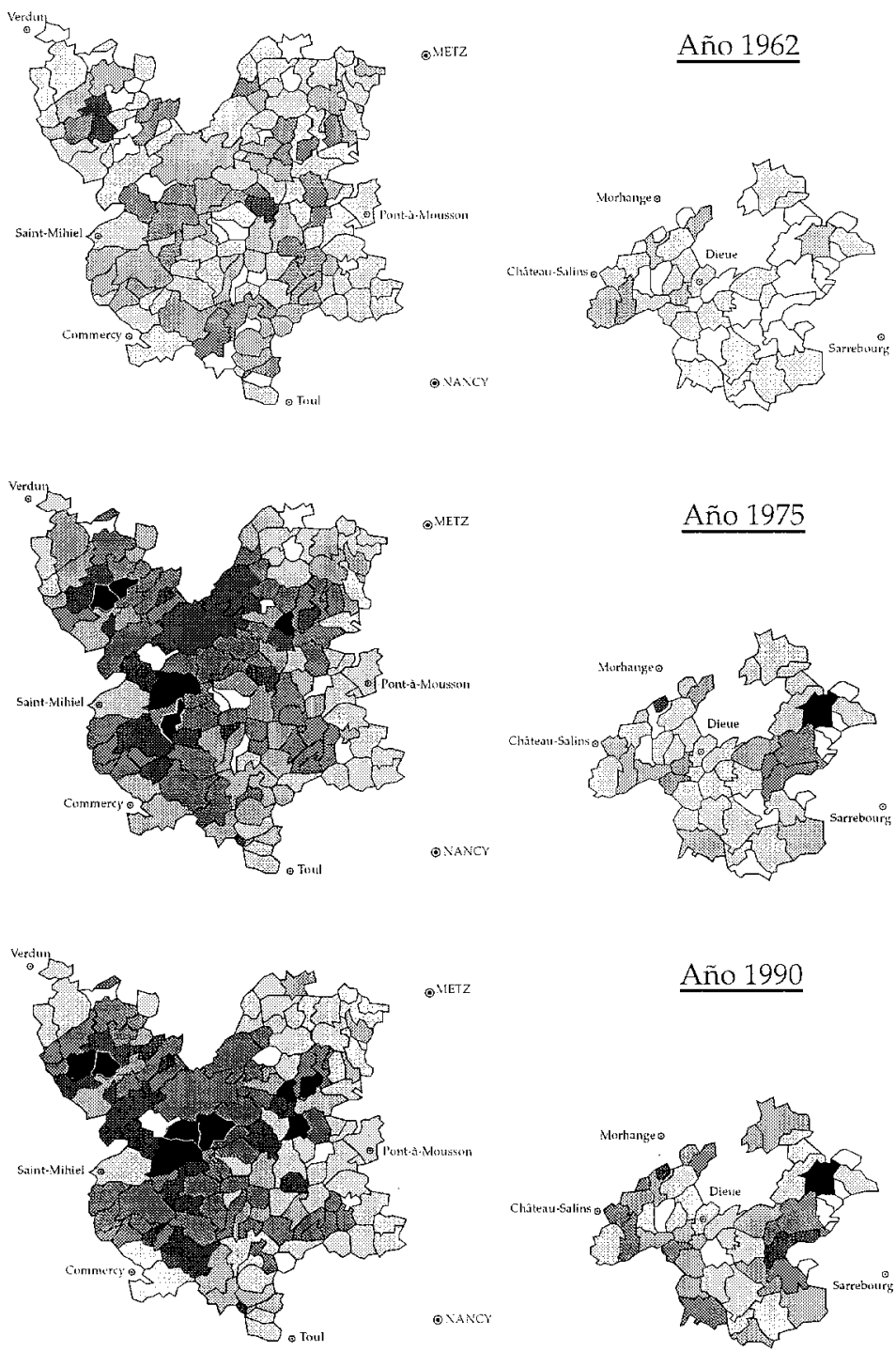

Año 1990
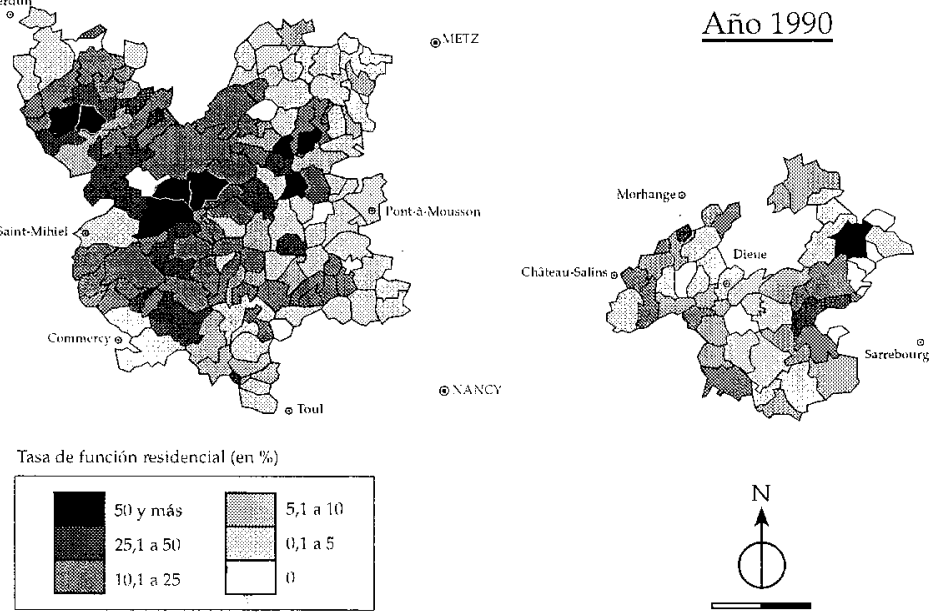

Fuente: INSEE. Elaboración propia. 
Figura 6.- Los complejos hidrorrecreativos y la oferta de camping en el Parque Natural Regional de Lorena

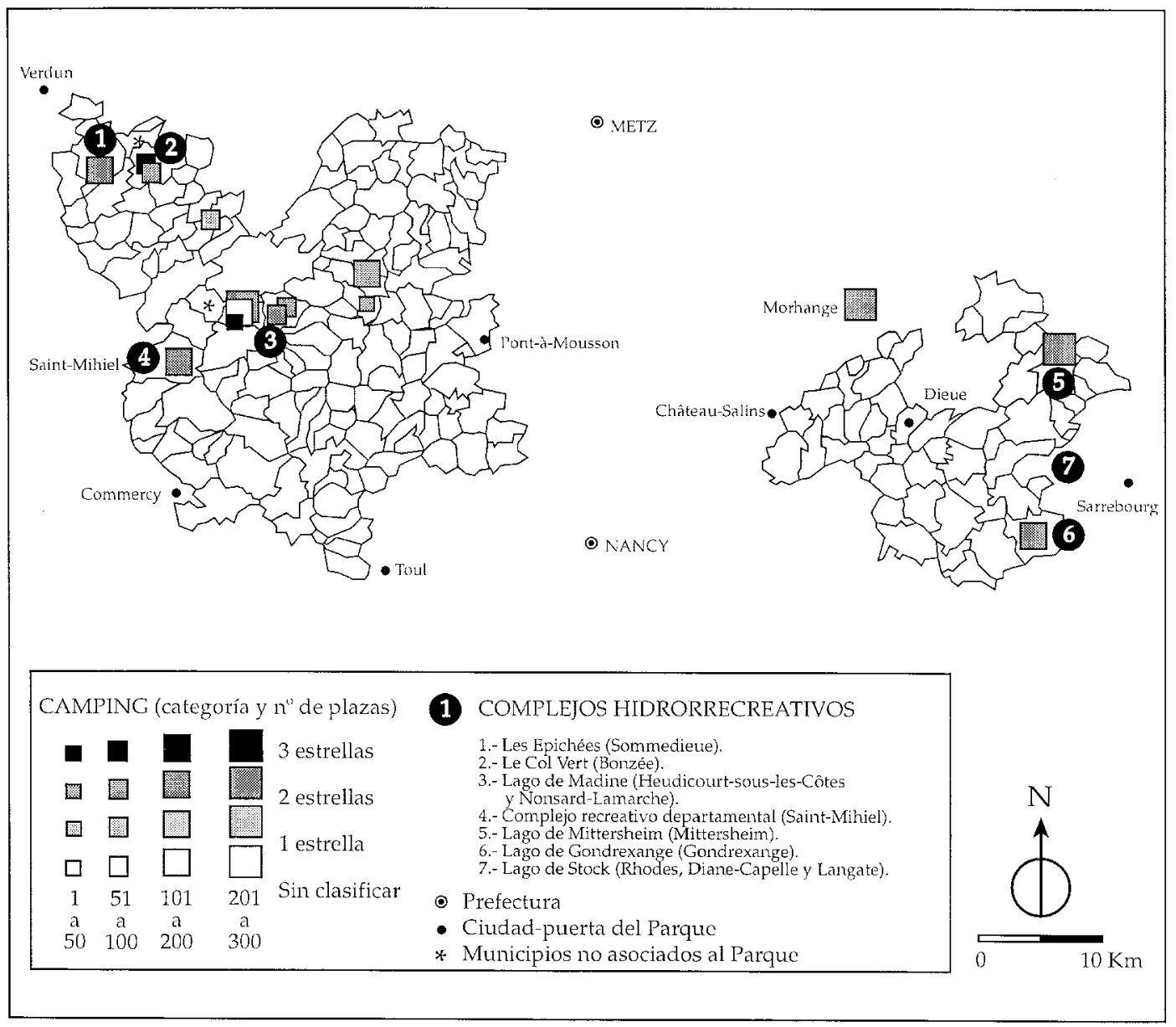

Fuente: Comités Turisticos Departamentales de Meurthe-et-Moselle, Meuse y Meurthe-et-Moselle. Elaboración propia. 
Figura 7.- La especialización de los municipios del Parque de Lorena según su tasa de función turística

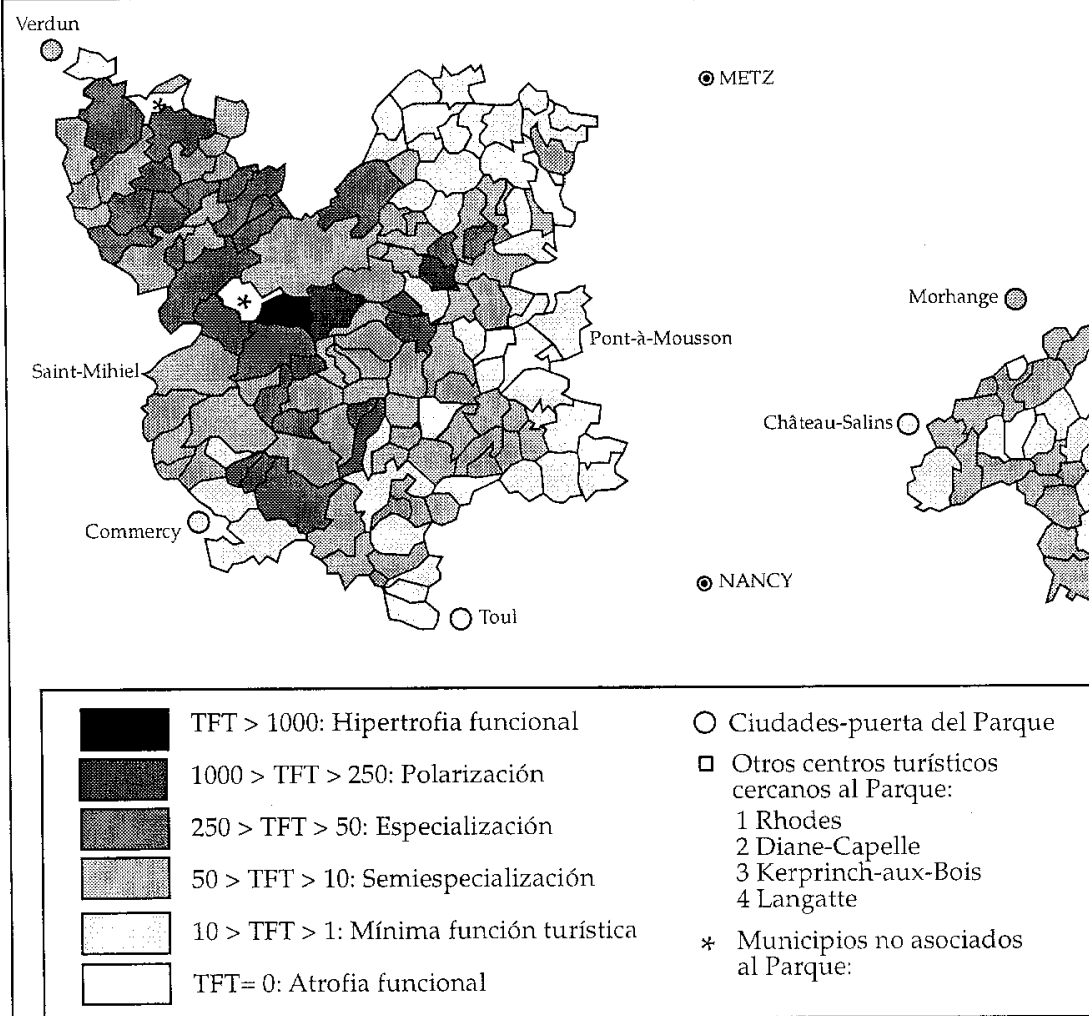

Fuente: Comités Turísticos Departamentales de Meurthe-et-Moselle, Meuse y Meurthe-et-Moselle; Federación Nacional de Gîtes de Francia. Elaboraciónpropia.

Las viviendas de turismo rural, integradas en la Féderation National des Gîtes de France, también tienen una representación importante dentro del Parque. En 1994 ofrecían plazas para unas 600 personas, poco más del $7 \%$ de la oferta regional, distribuyéndose entre 54 viviendas de turismo rural (gîtes ruraux), 14 casas de huéspedes (chambres d'hôtes), 7 albergues (gîtes de groupe ou d'etape), una casa rural para niños (gîtes d'enfants) y tres áreas naturales de acampada (aires naturelles de camping). Esta oferta, aunque reducida, es especialmente significativa por cuanto su localización responde a una lógica de difusión por todo el territorio. 
Con el fin de agotar las posibilidades analíticas en relación con la oferta de alojamiento, se ponderó el peso real de ésta respecto de la población permanente en los municipios del Parque, aplicando la tasa de función turística propuesta por DEFERT $(1967)^{15}$. La plasmación cartográfica de los resultados (Fig. 7) refleja las tendencias apuntadas al estudiar la residencia secundaria, bien que agudizadas por el efecto polarizador de los complejos hidrorrecreativos. No en vano, son los municipios que los soportan los que presentan una mayor especialización turística por la concentración y diversidad de la oferta (Nonsard y Héudicourt en el Lago de Madine, Mittersheim y Gondrexange junto a los lagos de su mismo nombre). Fuera del Parque, alrededor del lago de Stock, destacan los municipios de Rhodes, Diane-Capelle, Kerprich y Langatte, recogidos en este trabajo por su estrecha relación con el caso de Mittersheim.

\section{CONCLUSIONES}

El estudio de las acciones desarrolladas por el Parque y el análisis de los factores que explican el hecho turístico sobre su territorio permiten extraer las siguientes conclusiones:

1) El trabajo de los parques en materia de conservación y promoción no basta para explicar su transformación en destinos turísticos relevantes. Los parques se benefician de factores externos como son, por ejemplo, la proximidad a grandes ciudades, una localización litoral o la construcción de complejos recreativos.

2) En el caso del Parque de Lorena, su consolidación como destino turístico obedece a su proximidad a un área densamente urbanizada e industrializada. En este sentido, comparte rasgos periurbanos con otros parques franceses como son los de Brotonne, Haute-Vallé de Chevreuse, Lubéron, Montagne de Reims y Nord-Pas-deCalais.

3) La construcción de complejos hidrorrecreativos ha contribuido a incrementar la frecuentación, si bien los flujos turísticos que convergen hacia el Parque se han polarizado sobre aquéllos. Esto contribuye a consolidar la imagen del parque como lugar de ocio rururbano, en detrimento de su imagen de espacio natural de calidad.

4) La implantación de la oferta de alojamientos, especialmente de los campings y la residencia secundaria, se explica en razón del mismo proceso de polarización.

\footnotetext{
15 La tasa de función turística se formula de la siguiente forma: $\mathrm{TFT}_{\mathrm{i}}=\mathrm{CT}_{\mathrm{i}} \bullet 100 / \mathrm{P}_{\mathrm{i}}$; siendo $\mathrm{TFT}_{\mathrm{i}}$ la tasa de función turística de la localidad $\mathrm{i}, \mathrm{CT}_{\mathrm{i}}$ el número de camas turísticas en dicha localidad y $\mathrm{P}_{\mathrm{i}}$ la población permanente.
} 
5) Las acciones del parque encaminadas a la promoción del patrimonio natural y cultural (museos, senderos señalizados, centros de interpretación de la naturaleza, etc) han contribuido a consolidar flujos turísticos menores sobre su territorio de gran importancia por su carácter difuso e integración en el medio.

\section{BIBLIOGRAFIA}

BARBIER, B. (1965): "Méthode d'etude des résidences secondaires. L'exemple des Baises Alpes", Mediterranée, 2, 89-111.

CALLIZO, J. (1991): Aproximación a la geografía del turismo. Madrid: Síntesis.

CLARY, D. (1993): Le tourisme dans l'espace français. París: Masson.

CHADEFAUD, M. (1971): "Méthode d'analyse d'un flux touristique au niveau local et régional", Les Cahiers du Tourisme, serie C, 17.

DE MIGUEL, R. (1994): "Estado de la periurbanización en Francia: el caso de Nancy", Geographicalia, 31, 53-69.

DEFERT, P. (1967): "Le taux de fonction touristique. Mise au point et critique", Les Cahiers du Tourisne, serie C, 5 .

DEZERT, B. (1993): La France face ì l'ouverture eutropéenne. París: Masson.

DION, J. (1972): "Le Parc Naturel Régional de Lorraine", Reonue Géographique de l'Est, Tome 12, 4, 377-384.

FRECAUT, R. (1983): Géographie de la Lorraine. Nancy: Serpenoise.

FROMAGEAU, J. et GUTTINGER, P. (1993): Droit de l'environnement. París: Eyrolles.

LANNEAUX, M.A. et CHAPUIS, R. (1993): "Les parcs régionaux français", Amnales de Géograplite, $573,519-533$.

LAURENS, L. (1995): "Les parcs naturels, du concept à la pratique d'une agriculture environnementale", Amnales de Géographie, 584, 339-359.

RENUCCI, J. (1984): "Les résidences secondaires en France", Reoule de Géographie de Lyon, 1-2, 2940 .

REPARAZ, A. (1991): "De nouveaux rivages intérieurs. Les espaces de loisir liés aux plans d'eau artificiels en France", Actas del Coloquio de Lodz sobre turismo. 